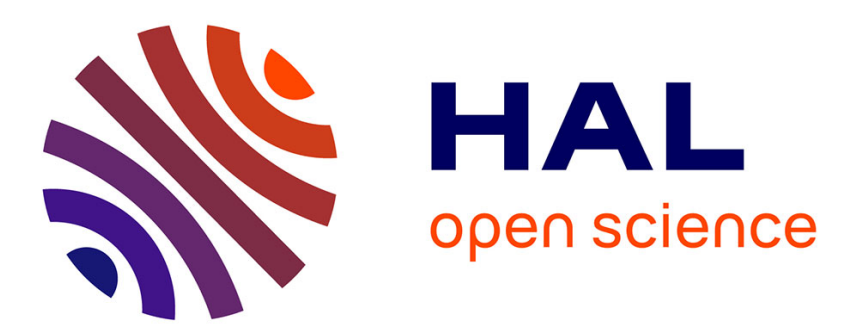

\title{
Utilisation du blé ou des pulpes de betteraves en rations complètes avec de l'ensilage de maïs chez les vaches laitières
}

\author{
A. Hoden, Catherine Hurtaud, B. Marquis, Luc Delaby
}

\section{- To cite this version:}

A. Hoden, Catherine Hurtaud, B. Marquis, Luc Delaby. Utilisation du blé ou des pulpes de betteraves en rations complètes avec de l'ensilage de maïs chez les vaches laitières. Productions Animales, 1990, 3 (4), pp.299-304. hal-00895913

\section{HAL Id: hal-00895913 https://hal.science/hal-00895913}

Submitted on 1 Jan 1990

HAL is a multi-disciplinary open access archive for the deposit and dissemination of scientific research documents, whether they are published or not. The documents may come from teaching and research institutions in France or abroad, or from public or private research centers.
L'archive ouverte pluridisciplinaire HAL, est destinée au dépôt et à la diffusion de documents scientifiques de niveau recherche, publiés ou non, émanant des établissements d'enseignement et de recherche français ou étrangers, des laboratoires publics ou privés. 
INRA Prod. Anim., 1990, $3(4), 299-304$
A. HODEN, Catherine HURTAUD

B. MARQUIS, L. DELABY

INRA Station de Recherches sur la Vache Laitière 35590 Saint-Gilles

\section{Utilisation du blé ou des pulpes de betteraves en rations complètes avec de l'ensilage de maïs chez les vaches laitières}

Dans les conditions habituelles de l'utilisation de l'ensilage de maïs, l'aliment concentré représente moins du quart de la ration totale chez les vaches laitières. La nature de la source énergétique utilisée (amidon ou parois) a alors peu d'influence sur les performances des animaux. Il importe de savoir s'il en est de même avec des rations plus extrêmes dont les teneurs en amidon et en cellulose brute varient dans de larges proportions?

\section{Résumé}

Deux rations complètes à base d'ensilage de maïs comportant un apport iso-énergétique (0,33 UFL/ $\mathrm{kg}$ MS) sous forme de blé ( $28 \%$ du mélange) ou de pulpes sèches de betteraves ( $33 \%$ du mélange) ont été comparées. Selon un schéma expérimental en inversion comportant 2 périodes de 6 semaines, 2 lots appariés de 13 vaches en phase décroissante de la lactation ont reçu à volonté chacune de ces rations.

Les niveaux d'ingestion ont été élevés et comparables pour les 2 régimes $(3,20 \mathrm{~kg}$ MS p. $100 \mathrm{~kg}$ de poids vif en moyenne), le blé et les pulpes représentant respectivement 5,2 et $6,0 \mathrm{~kg}$ MS par vache et par jour. Les teneurs en amidon des rations ont été respectivement de 39,6 et $20,2 \%$ et celles en cellulose brute de 14,3 et $18,8 \%$ pour les rations « blé " et "pulpes". Les apports énergétiques totaux (17 UFL en moyenne) ont été excédentaires $(+1,6$ UFL en moyenne) pour les 2 régimes mais surtout pour celui comportant la céréale ( $+2,3$ UFL).

Pour les 2 traitements " blé " et "pulpes ", les productions de lait, de matières grasses et de protéines ont été comparables et respectivement de 23,6 et $24,0 \mathrm{~kg}$, 943 et $953 \mathrm{~g}, 730$ et $735 \mathrm{~g}$ par jour. Le taux butyreux n'a pas été affecté par la nature des aliments complémentaires $(40,4$ et $40,1 \mathrm{~g} / \mathrm{kg})$, mais le taux protéique du lait a été significativement amélioré dans le cas de la ration comportant le blé $(31,3 \mathrm{~g}$ contre $31,0 \mathrm{~g} / \mathrm{kg})$. Au niveau technologique, ceci s'est traduit par un meilleur rendement fromager et une tendance à une amélioration des paramètres de la coagulation. Tous traitements confondus, les animaux ont gagné environ $250 \mathrm{~g} / \mathrm{j}$ sur l'ensemble de l'essai et les rations ont été correctement valorisées $(\mathbf{0 , 8 6}$ UFL/kg MS).

Malgré leurs caractéristiques de composition chimique très différentes, ces 2 types de rations n'ont que peu influencé les performances zootechniques. La légère influence positive de l'utilisation du blé par rapport aux pulpes est probablement à attribuer aux apports énergétiques supérieurs avec la céréale.
Le problème de l'utilisation des céréales par les vaches laitières et, d'une manière générale, celui de la nature des aliments complémentaires de la ration de base se pose depuis plusieurs années. Les résultats de nombreux essais montrent que les types d'aliments concentrés ont peu d'influence dans nos conditions d'emploi sur les performances des animaux (revues de Laurent 1988 et de Coulon et al 1989).

Les comparaisons des sources énergétiques à base d'amidon (céréales) et de parois (pulpes et sons) ont cependant dans la majorité des cas été réalisées avec des quantités d'aliments complémentaires relativement faibles (3 kg MS/ jour). Par ailleurs, peu d'études ont été effectuées dans les conditions françaises en utilisant la technique de la ration complète. Enfin, les résultats portant sur l'estimation de l'aptitude fromagère des laits sont peu nombreux alors que ce dernier aspect est devenu important dans le contexte technico-économique actuel. Or on peut penser que les modifications fermentaires au niveau du rumen (acides gras volatils) et l'éventuelle mise à la disposition de l'intestin de quantités différentes de glucose, induites par la nature des aliments complémentaires, ont une influence sur les synthèses de protéines dans la mamelle.

Durant l'hiver 1988-1989, ces différents aspects ont élé abordés à l'INRA de Rennes en 
comparant deux régimes distribués à volonté sous forme de rations complètes associant l'ensilage de maïs à du blé broyé grossièrement ou des pulpes de betteraves introduits à même quantité d'énergie. Les objectifs essentiels de cet essai étaient de mesurer les effets de la nature de la complémentation énergétique sur les performances des animaux et l'aptitude technologique des laits.

\section{Conditions expérimentales}

Selon un schéma expérimental en inversion, 2 lots appariés de 13 vaches, conduits sur paille, ont reçu à volonté durant 2 périodes de 6 semaines l'une des 2 rations complètes à base d'ensilage de maïs $(38 \%$ de MS, $48 \%$ de grains) comportant $28 \%$ de blé broyé grossièrement (grille de $10 \mathrm{~mm}$ ) ou $33 \%$ de pulpes sèches. Ces proportions avaient été calculées de façon à apporter la même quantité d'énergie (0,33 UFL/kg MS dans le mélange) sous forme de chacune des 2 sources. Chacun de ces 2 mélanges contenait également $7 \%$ de tourteaux de soja + colza protégés. Les 2 rations complètes ainsi constituées avaient des concentrations énergétiques de 1,02 et $0,97 \mathrm{UFL} / \mathrm{kg}$ MS respectivement pour les traitements «blé » et " pulpes " et une valeur azotée identique de $100 \mathrm{~g}$ de PDIE/kg MS. Par ailleurs, des quanti- tés constantes de divers compléments (urée, minéraux, vitamines) ont été ajoutées quotidiennement sur chacune de ces rations de façon à assurer la couverture des besoins d'environ $25 \mathrm{~kg}$ de lait par jour et à éviter tout trouble d'origine digestive (tableau 1).

Les critères d'appariement des animaux ont été le rang de lactation ( 9 primipares et 4 multipares par lot), le stade de lactation (76 $\pm 21 \mathrm{j}$ en début d'essai), la production laitière $(28,1 \pm$ $5,7 \mathrm{~kg})$, la composition du lait $(39,2 \pm 4,7 \mathrm{~g} / \mathrm{kg}$ de taux butyreux et $29,9 \pm 2,3 \mathrm{~g} / \mathrm{kg}$ de taux protéique) et le poids vif ( $585 \pm 55 \mathrm{~kg})$ observés au cours d'une pré-expérience de 3 semaines.

Les mesures individuelles des quantités de lait produites ont été effectuées chaque jour et la composition du lait (taux butyreux et de protéines) a été déterminée 3 jours par semaine ( 6 traites). Afin de mesurer l'aptitude technologique des laits, un échantillon représentatif issu d'une traite du matin de l'ensemble des vaches a été prélevé en dernière semaine de chacune des périodes pour les 2 traitements. Simultanément, en vue de mesurer la variabilité des réponses, 5 échantillons par traitement issus de laits de vaches à taux protéiques très différents (26,7 à $33,8 \mathrm{~g} / \mathrm{kg}$ en pré-expérience) ont été constitués. Tous ces prélèvements ont été écrémés immédiatement après la traite en vue de

Tableau 1. Utilisation comparée du blé et des pulpes de betteraves par les vaches laitières

A. Composition chimique et valeur nutritive des aliments (1)

\begin{tabular}{|c|c|c|c|c|c|c|c|c|}
\hline \multirow[b]{2}{*}{ Aliments } & \multicolumn{8}{|c|}{ Par kg de MS } \\
\hline & UFL & $\begin{array}{c}\text { PDIN } \\
\text { (g) }\end{array}$ & $\begin{array}{c}\text { PDIE } \\
\text { (g) }\end{array}$ & $\underset{\text { (g) }}{\text { MAT }}$ & $\begin{array}{l}\text { Cellulose } \\
\text { brute (g) }\end{array}$ & $\underset{(\mathrm{g})}{\text { Amidon }}$ & $\underset{(g)}{P}$ & $\begin{array}{l}\text { Ca } \\
\text { (g) }\end{array}$ \\
\hline Ensilage de maïs & 0,93 & 44 & 65 & 71 & 205 & 340 & 2,0 & 2,0 \\
\hline Tourteaux protégés & 1,13 & 394 & 365 & 496 & 82 & 30 & 8,7 & 4,4 \\
\hline Blé & 1,19 & 87 & 112 & 127 & 32 & 670 & 3,8 & 0,7 \\
\hline Pulpes de betteraves & 1,01 & 64 & 108 & 100 & 200 & 5 & 1,0 & 13,0 \\
\hline Urée & - & 1472 & - & 2875 & - & - & - & - \\
\hline
\end{tabular}

(1) Selon tables INRA, 1988 et dosage des matières azotées $(N \times 6,25)$ et cellulose brute.

B. Composition des rations complètes offertes à volonté

\begin{tabular}{|lcc|}
\hline \multicolumn{1}{|c|}{ Nature du concentré } & Blé & Pulpes \\
\hline Proportions (p. 100 de la MS) & & 60 \\
Ensilage de maïs & 65 & 33 \\
Blé ou pulpes & 28 & 7 \\
Tourteaux protégés & 7 & \\
\hline Apport systématique $(\mathrm{g} / \mathrm{j} /$ vache) & & 180 \\
Urée & 180 & 150 \\
Bicarbonate de sodium & 150 & 250 \\
Complément minéral 16-16 & 250 & 0 \\
Carbonate de calcium & 150 & 0 \\
\hline
\end{tabular}


réaliser les analyses et tests de laboratoire permettant d'apprécier l'aptitude à la coagulation (Formagraph) et le rendement fromager des laits (Vertes et al 1989). Les quantités ingérées ont été contrôlées quotidiennement pour chacun des lots et les animaux ont été pesés toutes les semaines.

L'ensemble des données individuelles a été traité par analyse de variance en utilisant la procédure GLM de SAS (SAS 1987) qui permet d'obtenir l'estimation de la moyenne des traitements en prenant en compte les effets des périodes et des individus.

\section{Résultats - Discussion}

\section{1 / Quantités ingérées}

Les quantités de matière sèche totale ingérées (tableau 2) des 2 rations complètes ont été comparables $(3,20 \mathrm{~kg}$ MS p. $100 \mathrm{~kg}$ de poids vif). Elles sont plus élevées d'environ $1 \mathrm{~kg}$ MS par jour et par vache par rapport aux capacités d'ingestion données par les Tables INRA (1988) à caractéristiques des animaux identiques (âge, niveau de production, poids vif). Elles correspondent cependant aux niveaux habituellement observés avec la technique de la ration complète (Augeard et al 1987; Hoden et Giger 1984).
Les proportions d'aliments complémentaires (blé ou pulpes + tourteaux) dans la ration totale ont été respectivement d'environ 34 et $39 \%$ pour les traitements "blé » et "pulpes». Simultanément, les teneurs en amidon ont été de 39,6 et $20,2 \%$ et celles en cellulose brute de 14,3 et $18,8 \%$.

L'ingestion supplémentaire dans le mélange de $0,8 \mathrm{~kg}$ MS de pulpes (6,0 vs 5,2 sous forme de blé) a entraîné une réduction de la consommation d'ensilage de maïs de $1,1 \mathrm{~kg}$ MS $[11,0$ vs 12,1 avec le blé). Ceci confirme l'encombrement important et au moins aussi élevé des pulpes par rapport au blé lorsqu'elles sont apportées sur des bases iso-énergétiques par rapport aux céréales (Coulon et al 1989 ; Faverdin et al 1990).

\section{2 / Performances et valorisation des rations}

Les productions n'ont pas été différentes selon les traitements et ont été en moyenne de $23,8 \mathrm{~kg}$ de lait, $948 \mathrm{~g}$ de matières grasses et $733 \mathrm{~g}$ de protéines (tableau 2). De même, le taux butyreux du lait a été élevé ( $>40 \mathrm{~g} / \mathrm{kg}$ ) et identique selon les régimes. Seul le taux protéique a été significativement plus élevé $(\mathrm{P}=$ 0,05 ) de $0,3 \mathrm{~g} / \mathrm{kg}$ avec la ration comportant le

Tableau 2. Effet de la nature du concentré énergétique sur les performances zootechniques des vaches laitières. (Résultats de $2 \times 13$ vaches $\times 6$ semaines/traitement)

\begin{tabular}{|c|c|c|c|}
\hline Nature du concentré & Blé & Pulpes & E.T.R. \\
\hline \multicolumn{4}{|l|}{ Quantités ingérées (kg MS) } \\
\hline Ensilage de maïs & 12,1 & 11,0 & \\
\hline Tourteaux protégés & 1,26 & 1,25 & \\
\hline Blé & 5,16 & - & \\
\hline Pulpes de betteraves & - & 6,02 & \\
\hline Urée - Minéraux & 0,69 & 0,55 & \\
\hline Total (\% poids vif) & 3,23 & 3,16 & \\
\hline Apports UFL corrigés (1) & 17,6 & 16,4 & \\
\hline Apports PDI (g) & 1715 & 1600 & \\
\hline MAT p.100 MS & 13,7 & 13,2 & \\
\hline Lait $(\mathrm{kg})$ & 23,6 & 24,0 & 0,89 \\
\hline Lait $4 \%(\mathrm{~kg})$ & 23,6 & 23,9 & 0,95 \\
\hline Matières grasses (g) & 943 & 953 & 43 \\
\hline Matières protéiques (g) & 730 & 735 & 23 \\
\hline Taux butyreux (p.1000) & 40,4 & 40,1 & 1,16 \\
\hline Taux protéique (p.1000) & $31,3^{* *}$ & 31,0 & 0,55 \\
\hline Poids vif $(\mathrm{kg})$ & 594 & 595 & 5,6 \\
\hline \multicolumn{4}{|l|}{ Bilan (apports-besoins) } \\
\hline UFL & $+2,3$ & $+0,9$ & \\
\hline PDI (g) & +195 & +65 & \\
\hline
\end{tabular}

\section{Les niveaux d'ingestion sont élevés et correspondent en moyenne à $3,20 \mathrm{~kg}$ $M S p 100 \mathrm{~kg}$ de poids vif pour les deux rations.}


blé (tableau 2). Ces données rejoignent celles obtenues dans d'autres comparaisons (Cotto et al 1988; Coulon et al 1989 ; Chenais et Kerouanton 1989 ; Laurent et Gardeur 1989).

Malgré la faible teneur en cellulose brute de la ration comportant le blé broyé grossièrement, les vaches n'ont pas présenté plus d'ennuis sanitaires (ni de chutes du taux butyreux) qu'avec le régime à base de pulpes. Mais les aliments complémentaires étaient apportés en mélange au fourrage (ration complète) et la ration comportait l'addition d'une substance tampon (bicarbonate de sodium). Cette observation confirme les données de nombreux autres essais (Coulon et al 1989; Laurent et Gardeur 1989).

Compte tenu du schéma expérimental en inversion et de la durée relativement courte de chacune des périodes ( 6 semaines), il est difficile d'estimer correctement la persistance de la production laitière et les variations réelles du poids vif selon le type de complémentation. Pour l'ensemble de l'essai (périodes $1+2$ ), la diminution de la production laitière n'a été que de $4 \%$ par mois et les reprises de poids vif corrigées des variations du contenu digestif (INRA, 1988) ont atteint environ $250 \mathrm{~g} / \mathrm{j}$.

Les apports nutritifs totaux ont été en moyenne pour les 2 traitements de 17,0 UFL (après déduction des effets d'interaction) et de $1660 \mathrm{~g}$ de PDI par jour. Ceux-ci ont été plus faibles d'environ 1, 2 UFL et $115 \mathrm{~g}$ de PDI pour la ration comportant les pulpes par rapport à celle à base de blé. Les apports d'énergie sous forme de chacun de ces 2 aliments complémentaires ont été pratiquement identiques et en moyenne de 6,1 UFL par jour, soit respectivement 35 et $37 \%$ des apports totaux des rations «blé » et «pulpes».

Tous traitements confondus, les vaches laitières ont été globalement sur-alimentées (apports corrigés - besoins entretien + production lait) sur l'ensemble de l'essai $(+1,6$ UFL et
+ $130 \mathrm{~g} \mathrm{PDI} / \mathrm{j})$ mais de façon plus importante lorsqu'elles ont reçu du blé (écart de $+1,4$ UFL et $130 \mathrm{~g}$ PDI par rapport aux pulpes). Ceci explique probablement l'excellente persistance de la production laitière et la bonne reprise de poids vif des vaches encore jeunes $(70 \%$ de primipares).

Par ailleurs, l'excès plus important d'énergie apportée aux vaches recevant la complémentation à base de blé doit probablement contribuer à expliquer le meilleur taux protéique observé par rapport aux pulpes (Laurent et Gardeur 1989 ; Rémond 1985). Ceci a été confirmé par des essais récents de Hurtaud et al (1990) montrant que les synthèses de protéines sont plus sensibles au niveau d'apport d'énergie qu'à sa nature.

La valorisation énergétique des 2 rations, calculée à partir des performances, est en moyenne de $0,86 \mathrm{UFL} / \mathrm{kg}$ MS en estimant le besoin de reprise de poids vif à $1 \mathrm{UFL} / \mathrm{j}$ compte tenu de la part représentée par la croissance des primipares $(3,5 \mathrm{UFL} / \mathrm{kg}$ gain contre 4,5 UFL pour la reconstitution des réserves corporelles - INRA, 1988). La même estimation effectuée à partir des apports nutritifs corrigés des interactions aboutit à la valeur de 0,89 UFL $/ \mathrm{kg}$ MS. Ces résultats sont satisfaisants compte tenu du niveau excédentaire des apports (INRA, 1988) et de la difficulté d'estimation des variations réelles du poids vif.

\section{3 / Caractéristiques biochimiques et aptitude fromagère du lait}

Les analyses biochimiques et les tests réalisés en fin de chacune des périodes confirment les modifications observées durant l'essai (tableau 3). L'apport de blé, associé à une sur-alimentation énergétique plus importante par rapport à la ration comportant les pulpes, ont eu pour conséquence d'augmenter le taux protéique $(+0,9 \mathrm{~g} / \mathrm{kg}-\mathrm{P}<0,05)$ et la teneur en caséine $(+0,5 \mathrm{~g} / \mathrm{kg}-\mathrm{N} . S$. $)$ du lait écrémé. Les quantités

Tableau 3. Effet de la nature du concentré énergétique sur la composition du lait écrémé. (Résultats de $2 \times 5$ vaches $\times 2$ périodes/traitement)

\begin{tabular}{|llll|}
\hline \multicolumn{1}{|c}{ Nature du concentré } & Blé & Pulpes & E.T.R. \\
\hline Composition du lait écrémé & & & \\
Azote total $(\mathrm{g} / \mathrm{kg}$ ) & $34,5^{* *}$ & 33,5 & 0,9 \\
Azote protéique $(\mathrm{g} / \mathrm{kg})$ & $32,9^{* *}$ & 32,0 & 0,9 \\
Azote caséinique $(\mathrm{g} / \mathrm{kg})$ & 26,6 & 26,1 & 0,8 \\
Protéines solubles $(\mathrm{g} / \mathrm{kg})$ & 5,61 & 5,57 & \\
$\alpha$ lactalbumine & 1,35 & 1,23 & 0,25 \\
$\beta$ lactoglobuline A & 1,68 & 1,87 & 0,30 \\
& 2,58 & 2,47 & 0,23 \\
Calcium total $(\mathrm{mg} / \mathrm{kg})$ & 1186 & $\mathbf{1 1 4 6}$ & 56,5 \\
Calcium soluble $(\mathrm{mg} / \mathrm{kg})$ & 326 & 322 & 20,6 \\
Calcium colloïdal $(\mathrm{mg} / \mathrm{kg})$ & 860 & 824 & 48,1 \\
\hline
\end{tabular}

Niveau de signification entre moyennes ajustées : ${ }^{*} \mathrm{P}<0,05$. 
Tableau 4. Effet de la nature du concentré énergétique sur l'aptitude à la coagulation et l'aptitude fromagère des laits. (Résultats de $2 \times 5$ vaches $\times 2$ périodes/traitement)

\begin{tabular}{|lccc|}
\hline \multicolumn{1}{|c}{ Nature du concentré } & Blé & Pulpes & E.T.R. \\
\hline « Rendements fromagers » (\%) & & & \\
« Rendement » en frais & $18,5^{* *}$ & 17,6 & 1,1 \\
« Rendement » en azote & 78,3 & 78,0 & 2,0 \\
« Rendement » en MS & $44,5^{\star}$ & 42,9 & \\
\hline Aptitude à la coagulation & & & 0,03 \\
(Formagraph) & & & 2,1 \\
* Au pH initial du lait & $6,66^{*}$ & 6,68 & 1,5 \\
Temps de prise (min) & 19,1 & 20,6 & 6,3 \\
Temps de raffermissement (min) & $9,4^{*}$ & 10,7 & 0,1 \\
Indice de fermeté (mm) & 24,6 & 20,3 & \\
Indice de vitesse & 2,2 & 2,1 & 2,4 \\
* Au pH standardisé du lait (6,6) & & & 1,4 \\
Temps de prise (min) & 14,1 & 13,9 & 6,4 \\
Temps de raffermissement (min) & 6,7 & 7,1 & 0,1 \\
Indice de fermeté (mm) & 38,3 & 35,9 & 2,2 \\
Indice de vitesse & $2,3^{*}$ & & \\
\hline
\end{tabular}

Niveau de signification entre moyennes ajustées : ${ }^{*}-\mathrm{P}<0,1 ;{ }^{* *}=\mathrm{P}<0,05$.

de protéines solubles ont été moins modifiées (N.S.) sous l'effet de l'apport de blé $(+0,12$ $\mathrm{g} / \mathrm{kg}$ de l'alpha-lactalbumine et $-0,08 \mathrm{~g} / \mathrm{kg}$ de la béta-lactoglobuline). De même, l'aliment concentré à base de blé a provoqué une augmentation non significative de la teneur en calcium total ( $+40 \mathrm{mg} / \mathrm{kg}$ ) due essentiellement à une élévation de la teneur en calcium colloïdal (+ $36 \mathrm{mg} / \mathrm{kg}$ ).

Simultanément, ces modifications ont eu des répercussions sur les propriétés technologiques des laits, en particulier sur le rendement (tableau 4). En effet, l'apport de blé a entraî̀né une augmentation du « rendement fromager» en frais $(+0,9 \%-\mathrm{P}<0,05)$ et en matière sèche $(+1,6 \%-$ N.S. $)$. L'aptitude à la coagulation a été relativement peu modifiée par l'apport de blé. Au pH initial du lait, les temps de prise et de raffermissement sont diminués (-1,5 min environ) tandis que l'indice de fermeté est augmenté (+ 4,3 mm - N.S.). Au pH standardisé du lait, l'apport du blé n'a entraîné aucune amélioration significative de l'aptitude à la coagulation. Seules certaines tendances se dégagent telles que la diminution du temps de raffermissement (- 0,4 min - N.S.), l'augmentation de l'indice de fermeté $(+2,4 \mathrm{~mm}-\mathrm{N}$.S.) et l'accroissement du temps de prise $(+0,2 \mathrm{~min}-$ N.S.), effet plutôt négatif.

\section{Conclusion}

Les rations complètes à base d'ensilage de maïs comportant plus du tiers de leur énergie sous forme de blé ou de pulpes sèches de betteraves ont permis globalement les mêmes performances zootechniques et ont été bien valorisées. Ces 2 régimes n'ont que peu modifié l'en- semble des paramètres mesurés comparativement à leurs différences de composition chimique $(19,4$ points de teneur en amidon et 4,5 points en cellulose brute).

Les niveaux d'ingestion ont été élevés et les animaux ont été sur-alimentés, surtout avec la ration comportant le blé. Dans ces conditions, la teneur en azote total, les fractions azotées (protéines, caséines) ainsi que les teneurs en calcium (total et colloïdal) du lait ont été sensiblement améliorées lorsque les vaches recevaient le blé. Au niveau technologique, ceci s'est traduit essentiellement par un meilleur rendement fromager et une tendance à une amélioration des paramètres de la coagulation.

Ces résultats sont en accord avec ceux déjà obtenus d'une part avec la technique des rations complètes (Hoden et Giger 1984 ; Augeard et al 1987) et d'autre part sur la nature des aliments complémentaires utilisés (Cotto et al 1989 ; Faverdin et al 1990). L'influence légèrement positive de l'utilisation du blé par rapport aux pulpes est probablement à attribuer aux apports énergétiques supérieurs et/ou à la mise à la disposition plus importante de nutriments (glucose, acides aminés) au niveau de la mamelle (Rémond 1985 ; Rulquin 1987 ; Hurtaud et al 1990) avec la céréale.

\section{Remerciements}

Nous tenons à remercier I'Union Laitière Normande (50890 Conde-sur-Vire) de son concours technique qui a permis la réalisation de cette étude.
Le taux protéique

plus élevé et l'amélioration sensible de l'aptitude fromagère des laits dans le cas de la ration comportant le blé doivent être reliés à une sur-alimentation énergétique importante avec la céréale. 


\section{Références bibliographiques}

AUGEARD Ph., BAZIN S., CHENAIS F., GIRARD P., WEISS Ph., 1987. Rations complètes ou distribution individuelle de concentré. Document ITEB n ${ }^{\circ} 87043,84$ p.

CHENAIS F., KEROUANTON J., 1989. Quel concentré pour les vaches laitières? Pulpes ou céréales. Document ITEB-EDE n" 89121, $44 \mathrm{p}$

COTTO Geneviève, LAGRANGE Cécile, CHENAIS F., LAURENT F., WEISS Ph., 1988. Pour les vaches laitières quel concentré? Pulpes ou céréales. L'utilisation des céréales par les vaches laitières. ITEB - Paris 7. juin 1988.

COULON J.B., FAVERDIN Ph., LAURENT F., COTTO Geneviève, 1989. Influence de la nature de l'aliment concentré sur les performances des vaches laitières. INRA Prod Anim., 2, 47-53.

FAVERDIN Ph. DULPHY J.P., COULON J.B, VERITE R, GAREL J.P., ROUEL J., MARQUIS B., 1990. Substitution of roughage by concentrates for dairy cows. Livestock Production Science (sous presse).

HODEN A., GIGER Sylvie, 1984. Les rations complètes pour vaches laitières. Revue bibliographique. Bull. Tech. CRZV Theix, INRA, 57, 45-50.

HURTAUD Catherine, RULQUIN H., VERITE R., 1990. Effects on milk yield and composition of infusions of volatile fatty acids and caseinate into the digestive tract of dairy cows. VIe Journées de Recherches sur l'Alimentation et la Nutrition des Herbivores. 21-22 Mars 1990 INA-PG Paris.
HURTAUD Catherine, RULQUIN H., VERITE R., 1990 Effects on milk vield and composition of infusions of different level and nature of energy into the digestive tract of dairy cows. VI" Journées de Recherches sur l'Alimentation et la Nutrition des Herbivores. 21-22 Mars 1990 INA-PG Paris

INRA, 1988. Alimentation des bovins, ovins et caprins Ed. R. JARRIGE, INRA Publications, Route de St-Cyr 78000 Versailles.

LAURENT F., 1988. Utilisation du blé et des céréales dans la ration des vaches laitières. Ann. Zootech., 37, 117-132.

LAURENT F., GARDEUR J.N., 1989. Effet de la nature et du niveau d'apport en aliment concentré sur les performances des vaches laitières recevant une ration à base d'ensilage de maïs. Ann. Zootech., 38, 247-258.

REMOND B., 1985. Influence de l'alimentation sur la composition du lait de vache. II. Taux protéique : facteurs généraux. Bull. Tech. CRZV Theix, INRA, 62, 53-67.

RULOUIN H., 1987. Détermination de certains acides aminés limitants chez la vache laitière par la méthode des administrations post-ruminales. Reprod. Nutr. Dévelop. 27 (1B), 299-300.

SAS INSTITUTE INC., 1987. SAS/STAT. Guide for personnal computer, Version 6, Edition Cary N.C.

VERTES Catherine, HODEN A., GALLARD Y., 1989. Effet du niveau d'alimentation sur la composition chimique et la qualité fourragère du lait de vaches Holstein et Normandes. INRA Prod. Anim., 2, 89-96,

\section{Summary}

The use of a combined wheat or beet pulp and maize silage diet in dairy cattle.

Two complete diets based on maize silage including an iso-energetic contribution $[0.33$ UFL $/ \mathrm{kg} \mathrm{MS)} \mathrm{in} \mathrm{the} \mathrm{form} \mathrm{of} \mathrm{either} \mathrm{wheat} \mathrm{(} 28 \%$ of the mixture) or dry beet pulp (33\%) were compared. For two periods of six weeks, two groups of 13 cows each, in the declining phase of lactation, were observed. One group received the wheat diet and the other the beet pulp diet.

Ingestion levels increased and were comparable for the two diets $(3.20 \mathrm{~kg} M S / 100 \mathrm{~kg}$ live weight, on average), wheat $(5.2 \mathrm{~kg} \mathrm{MS} /$ cow/day) and beet pulp (6.0 kg MS), representing, respectively, 27 and $32 \%$ of the total diet. Starch content in the diets were respectively 39.6 and $20.2 \%$ and that of crude cellulose 14.3 and $18.8 \%$ for wheat and beet pulp diets. The total energetic values (17 UFL on average) were in excess of this for both diets $(+1.6$ UFL on average), but mainly for the cereal ( +2.3 UFL)

With both wheat and beet pulp diets, milk production, fat content and protein content were comparable, respectively, 23.6 and 24.0 $\mathrm{kg}, 943$ and $953 \mathrm{~g}, 730$ and $735 \mathrm{~g}$ per day. The fat content was not affected by the nature of the complementary feed $(40.4$ and $40.1 \mathrm{~g} / \mathrm{kg}$ ) but the protein content of the milk significantly increased with the wheat diet $[31.3$ compared to $31.0 \mathrm{~g} / \mathrm{kg}$ ). Technologically, this represents a better cheese yield and improved the coagulation properties. For both treatments the animals gained about 250 g/day throughout the experimental trial and the diets were correctly estimated $(0.86 \mathrm{UFL} / \mathrm{kg}$ MS).

Despite the fact that the two diets were very different chemically, their effect on production was limited. The slight increase with the wheat diet, was probably due to the greater energy content of the cereal.

HODEN A., HURTAUD Catherine, MARQUIS B., DELABY L. 1990. Utilisation du blé ou des pulpes de betteraves en rations complètes avec de l'ensilage de maïs chez les vaches laitières. INRA Prod. Anim., 3 , (4), 299-304. 